Jinks write too much about inbred lines and their own data, but in this book their analysis gives equal weight to studies of inbred lines and randomly mating populations. Few programmes have explored the genetics of quantitative variation with such precision as that in Birmingham. The species selected for study have been especially amenable to genetic manipulation through breeding, randomization was conducted at the level of individual plants rather than plots and, above all, the authors have data on the same material going back over 30 years in many cases.

Researchers in genetics and the social sciences will discover that the genetic basis of individual differences is more complex than is presupposed in many models for human variation. The strength of the approach to variation developed by Mather and Jinks lies in representing individual genes algebraically and tracing their cumulative and interactive effects on a continuous phenotype. The models extend readily to include genotypeenvironment interaction and the effects of genes on environment which are often discounted arbitrarily in human studies. In many respects, therefore, Mather and Jinks pre-empt much of the recent work on non-genetic transmission. One factor, however, prevents the whole-hearted application of the biometrical approach to human populations. The models they develop begin at the genotype and work

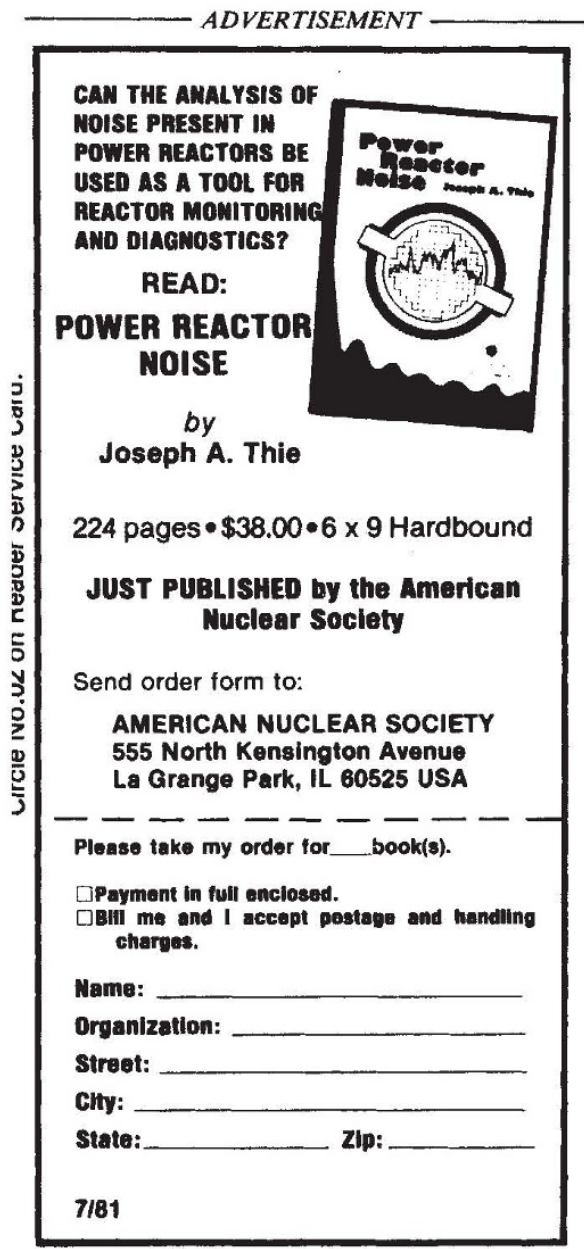

outwards. The approach is powerful when the genetic structure of a population can be manipulated by breeding and when interactions between phenotypes can be minimized by randomization. In human populations, however, the consequences of assortative mating and cultural inheritance may depend on social interactions at the phenotypic level and are difficult to represent by the cumulative finite effects of many discrete loci. For this reason, the treatment of assortative mating requires distinct methods which have still to be reconciled with the biometrical approach. Similarly, although Mather and Jinks trace the environmental consequences of genetic differences such as those of the maternal genotype, models of family resemblance for cultural traits ultimately require series which do not end at the parental genotype.

Although the book will help others design, analyse and interpret their own experiments, it is not just a "cookbook". It presents the single most compelling body of evidence to remind all who study variation in plants, animals or man that genetic effects on continuous variation are inherently subtle and that the failure of studies to resolve them says more about basic limitations in the design and analysis of investigations than the underlying simplicity of genetic effects.

The significance of the book lies in the rigorous model-building and data collection which lie behind the authors' attempt to analyse continuous variation. Mather and Jinks's own thorough statement of the theory of multifactorial inheritance and its implications for the analysis of phenotypic variation is based on two generations of painstaking work. Such experience cannot be won cheaply. The opportunity to share the authors' insight will be the lasting value of their work for any investigator of the new generation with the stamina to follow it.

Lindon J. Eaves is Distinguished Professor of Human Genetics at the Medical College of Virginia, Richmond, Virginia.

\section{Being sure of Shell}

\section{Bruce Sellwood}

Geological Atlas of Western and Central Europe. By Peter A. Ziegler. Pp.130. (Elsevier Scientific: 1982.) Dfl.160, \$68.

AS WELL as being tremendous fun to draw, palaeogeographical maps are essential to the exploration geologist. Such maps aid the delineation of sedimentary basins and are a vital tool to the extractive geological industries. In addition to the former position of shore lines, such maps should also show the nature of the deposited sediment within the basin and its thickness.
Of course, the accuracy of a palaeogeographical map depends to a large extent on the nicety of regional geological correlation and the quality of information available. The data points provided - or implied - on a such a map are like the data points on a graph. They allow the reader to assess the validity of the interpretation provided by the map itself.

The Geological Atlas of Western and Central Europe provides an overview of the tectonic and stratigraphic framework and a summary of European evolution from the Late Silurian onwards, a period of about $400 \mathrm{Myr}$. It is a lavishly illustrated tour de force and Peter Ziegler is to be congratulated on the production and presentation of a fine working document. Shell too deserve considerable praise in permitting the release of their collected regional data. The maps (40 full-colour enclosures) and 29 line drawings provide thought-provoking frames of reference for the exploration geologist and academic stratigrapher alike.

In a work of this breadth there are inevitably points of criticism. The maps, for instance, are largely devoid of data points, it being suggested that this is because of confidentiality and the constraints of space. However, two transparent overlays giving the outline of Europe are provided and it would have been a relatively simple task to locate released wells on these.

The text is a narrative to the coloured maps, but as such it does not provide sufficient basic information. Thus speculations embodying vastly broad concepts (e.g. convergence and collision in trench-arc systems) are sometimes left as ex cathedra statements unqualified by even a supporting source reference. (To be fair, however, there are over 650 references, all essential, and so the book provides a valuable bibliographic overview of European evolution.) I also found the time-frame of some maps misleading. Instead of providing maps over as precise as possible a time-period, some have been needlessly generalized. This is particularly true within the Mesozoic where, for example, one map covers the bulk of the Lower and part of the Middle Jurassic (a 27 Myr period), another encompasses the entire Upper Jurassic (a 19 Myr period) and yet another embodies the Upper Cretaceous to lowest Tertiary (about 37.3 Myr). Such maps are confusing at best and misleading at worst, the equivalent of attempting to capture all England's Tudor monarchs in one single photofit image!

Notwithstanding these criticisms the two-volume compilation embodies a colossal labour by a geologist of great talent. The Geological Atlas will be a valuable and provocative base for future research - and it comes at a very reasonable price.

Bruce Sellwood is Lecturer in Geology at the University of Reading. 